\title{
Hamiltonian structures for general PDEs
}

\author{
P. Kersten ${ }^{1}$, I.S. Krasil'shchik ${ }^{2}$, A.M. Verbovetsky ${ }^{2}$, and R. Vitolo ${ }^{4}$ \\ 1 P.H.M. Kersten, University of Twente, Postbus 217, 7500 AE Enschede, the Netherlands \\ kerstenphm@ewi.utwente.nl \\ 2 I.S. Krasil'shchik, A.M. Verbovetsky, Independent University of Moscow, B. Vlasevsky 11, \\ 119002 Moscow, Russia \\ josephk@diffiety.ac.ru,verbovet@mccme.ru \\ 3 R. Vitolo, Dept. of Mathematics “E. De Giorgi”, Università del Salento, via per Arnesano, \\ 73100 Lecce, Italy \\ raffaele.vitolo@unile.it
}

Summary. We sketch out a new geometric framework to construct Hamiltonian operators for generic, non-evolutionary partial differential equations. Examples on how the formalism works are provided for the KdV equation, Camassa-Holm equation, and Kupershmidt's deformation of a bi-Hamiltonian system.

\section{Introduction}

In this short paper we will discuss the following question: What happens to a Hamiltonian operator of an evolution system if we change coordinates so that the system becomes non-evolution?

Using the traditional definition of a Hamiltonian structure one cannot answer this question, since the definition is tied to evolution form of the system at hand. However, first, not all equations have a natural evolution form, and, second, an evolution form of a system of equations is not unique. Let us consider some examples.

Example $1(K d V)$. It is well known that the $\mathrm{KdV}$ equation $u_{t}=u_{x x x}+6 u u_{x}$ has two compatible Hamiltonian operators:

$$
A_{1}=D_{x}, \quad A_{2}=D_{x x x}+4 u D_{x}+2 u_{x},
$$

so that the equation can be written in the following ways:

$$
u_{t}=u_{x x x}+6 u u_{x}=D_{x} \frac{\delta}{\delta u}\left(u^{3}-u_{x}^{2} / 2\right)
$$

\footnotetext{
This work was supported in part by the NWO-RFBR grant 047.017.015 (PK, IK, and AV), RFBR-Consortium E.I.N.S.T.E.IN grant 06-01-92060 (IK, AV, and RV) and RFBR-CNRS grant 08-07-92496 (IK and AV).
} 


$$
=\left(D_{x x x}+4 u D_{x}+2 u_{x}\right) \frac{\delta}{\delta u}\left(u^{2} / 2\right),
$$

where $\delta / \delta u$ denotes the Euler operator (the variational derivative) and is applied to the two Hamiltonian densities.

Let us introduce new dependent variables $v$ and $w$ and rewrite the $\mathrm{KdV}$ equation in the form

$$
u_{x}=v, \quad v_{x}=w, \quad w_{x}=u_{t}-6 u v .
$$

In the new coordinates, the KdV still has an evolutionary form, but with respect to another independent variable ( $x$ instead of $t$ ). A natural question arises then: Is the $\mathrm{KdV}$ equation in the form (2) Hamiltonian? An affirmative answer to this question was obtained by Tsarev in [9]. He proved that transformations of the type (2) preserve the Hamiltonian property of all evolution systems for which the Cauchy problem is solvable. Our approach is very different from Tsarev's one. Below we explain why this fact holds true for all transformations of variables and without the assumption on the Cauchy problem. We will also show how to compute the Hamiltonian structure in new coordinates. For the above example the answer is the following:

$$
\begin{aligned}
\left(\begin{array}{l}
u \\
v \\
w
\end{array}\right)_{x} & =\left(\begin{array}{ccc}
0 & -1 & 0 \\
1 & 0 & -6 u \\
0 & 6 u & D_{t}
\end{array}\right)\left(\begin{array}{c}
\delta / \delta u \\
\delta / \delta v \\
\delta / \delta w
\end{array}\right)\left(u w-v^{2} / 2+2 u^{3}\right) \\
& =\left(\begin{array}{ccc}
0 & -2 u & -D_{t}-2 v \\
2 u & D_{t} & -12 u^{2}-2 w \\
-D_{t}+2 v & 12 u^{2}+2 w & 8 u D_{t}+4 u_{t}
\end{array}\right)\left(\begin{array}{l}
\delta / \delta u \\
\delta / \delta v \\
\delta / \delta w
\end{array}\right)\left(-3 u^{2} / 2-w / 2\right)
\end{aligned}
$$

Example 2 (Camassa-Holm equation). Camassa and Holm have written their equation $u_{t}-u_{t x x}-u u_{x x x}-2 u_{x} u_{x x}+3 u u_{x}=0$ in a bi-Hamiltonian form by introducing the new variable $m=u-u_{x x}$. The equation now takes the form

$$
m_{t}=-u m_{x}-2 u_{x} m=B_{1} \frac{\delta \mathscr{H}_{1}}{\delta m}=B_{2} \frac{\delta \mathscr{H}_{2}}{\delta m}
$$

with

$$
\begin{aligned}
& B_{1}=-\left(m D_{x}+D_{x} m\right), \quad \mathscr{H}_{1}=\frac{1}{2} \int m u d x, \\
& B_{2}=D_{x}^{3}-D_{x}, \quad \mathscr{H}_{2}=\frac{1}{2} \int\left(u^{3}+u u_{x}^{2}\right) d x .
\end{aligned}
$$

Note that $\mathscr{H}_{1}$ and $\mathscr{H}_{2}$ are viewed as functionals in $m$ and $u$, but not in $u$ solely. To get rid of $m$, one is forced to assume that $u=\left(1-D_{x}^{2}\right)^{-1} m$ in the Hamiltonian densities. The use of the inverse of the operator $1-D_{x}^{2}$ is not elegant from mathematical viewpoint. We will find a bi-Hamiltonian structure for the Camassa-Holm equation written in the initial non-evolution form and thus get rid of the term $\left(1-D_{x}^{2}\right)^{-1}$. 
Example 3 (Kupershmidt deformation). Consider a bi-Hamiltonian evolution system of equations $u_{t}=f\left(t, x, u, u_{x}, u_{x x}, \ldots\right), u$ and $f$ being vector functions, with compatible Hamiltonian operators $A_{1}$ and $A_{2}$ and a Magri hierarchy of conserved densities $H_{1}, H_{2}, \ldots$

$$
D_{t}\left(H_{i}\right)=0, \quad A_{1} \frac{\delta H_{i}}{\delta u}=A_{2} \frac{\delta H_{i+1}}{\delta u} .
$$

In [8], Kupershmidt defined what he called the nonholonomic deformation of the above system:

$$
u_{t}=f-A_{1}(w), \quad A_{2}(w)=0 .
$$

We call system (5) the Kupershmidt deformation of the system $u_{t}=f$. The motivating example of this construction is the so-called KdV6 equation (see [4])

$$
u_{t}=u_{x x x}+6 u u_{x}-w_{x}, \quad w_{x x x}+4 u w_{x}+2 u_{x} w=0
$$

which is the Kupershmidt deformation of the KdV equation. The authors of [4] have shown that the KdV6 passes the Painlevé test and conjectured that the system is integrable. Kupershmidt, in [8], found a hierarchy of conservation laws of the KdV6 as a particular case of the following general fact.

Theorem (Kupershmidt). Let $u_{t}=f$ be an evolution bi-Hamiltonian system, with $A_{1}, A_{2}$ being the corresponding Hamiltonian operators. If this equation has a Magri hierarchy of conserved densities $\frac{d H_{i}}{d t}=0, A_{1} \frac{\delta H_{i}}{\delta u}=A_{2} \frac{\delta H_{i+1}}{\delta u}$ then $H_{1}, H_{2}, \ldots$ are conserved densities for (5).

Proof.

$$
\begin{aligned}
\frac{d H_{i}}{d t}=\left\langle\frac{\delta H_{i}}{\delta u}, f+A_{1}(w)\right\rangle & =\left\langle-A_{1} \frac{\delta H_{i}}{\delta u}, w\right\rangle \\
& =\left\langle-A_{2} \frac{\delta H_{i+1}}{\delta u}, w\right\rangle=\left\langle\frac{\delta H_{i+1}}{\delta u}, A_{2}(w)\right\rangle=0 .
\end{aligned}
$$

Kupershmidt also conjectured that $H_{1}, H_{2}, \ldots$ commute in some sense so that the KdV6 is indeed integrable. Below we will see that this is true and, moreover, system (5) is bi-Hamiltonian.

Our framework to study Hamiltonian structures for general PDEs is the geometry of jet spaces and differential equations. We assume the reader to be familiar with the geometric approach to differential equations and hence we include only the notation and the coordinate descriptions in the next section. We refer the reader to the books [1, 6] for further information.

\section{Notation: infinite jets and differential equations}

In what follows everything is supposed to be smooth. 
We denote an infinite jet space by $J^{\infty}$. This can be the space of jets of submanifolds, maps, sections of a bundle, and so on, and it is not important to us here. Coordinates on $J^{\infty}$ are $x_{i}$ (independent variables, $i=1, \ldots, n$ ) and $u_{\sigma}^{j}$ (dependent variables, $j=1, \ldots, m, \sigma$ being multi-indices).

The formulas

$$
D_{i}=\frac{\partial}{\partial x_{i}}+\sum_{j, \sigma} u_{\sigma i}^{j} \frac{\partial}{\partial u_{\sigma}^{j}}
$$

provide expressions for the total derivatives. The vector fields $D_{i}$ span the Cartan distribution on $J^{\infty}$. To every vector function on $J^{\infty}$, there corresponds the evolutionary field

$$
E_{\varphi}=\sum_{j, \sigma} D_{\sigma}\left(\varphi^{j}\right) \frac{\partial}{\partial u_{\sigma}^{j}}
$$

The matrix differential operator

$$
\ell_{f}=\left\|\sum_{\sigma} \frac{\partial f^{i}}{\partial u_{\sigma}^{j}} D_{\sigma}\right\|
$$

is the linearization of a vector function $f$. It is defined by the formula $\ell_{f}(\varphi)=E_{\varphi}(f)$. The linearization is a differential operator in total derivatives; we shall call such operators $\mathscr{C}$-differential operators.

The coordinate expression for the adjoint $\mathscr{C}$-differential operator is

$$
\Delta^{*}=\left\|\sum_{\sigma}(-1)^{|\sigma|} D_{\sigma} a_{\sigma}^{j i}\right\|
$$

if $\Delta=\left\|\sum_{\sigma} a_{\sigma}^{i j} D_{\sigma}\right\|$.

Let $F_{k}\left(x_{i}, u_{\sigma}^{j}\right)=0, k=1, \ldots l$, be a system of differential equations. Then the relations $F=\left(F_{1}, \ldots, F_{l}\right)=0$ together with $D_{\sigma}(F)=0$ define its infinite prolongation $\mathscr{E} \subset J^{\infty}$. For the sake of brevity we shall call the infinite prolongation of a system of differential equations the equation. The operator $\ell_{\mathscr{E}}=\left.\ell_{F}\right|_{\mathscr{E}}$ is the linearization of the equation $\mathscr{E}$.

In this paper, we only consider equations $\mathscr{E}$ whose linearization $\ell_{\mathscr{E}}$ is normal in the following sense.

Definition 1. A $\mathscr{C}$-differential operator $\nabla$ called normal if the compatibility operators for both $\nabla$ and $\nabla^{*}$ are trivial. In other words, if there exists a $\mathscr{C}$-differential operator $\Delta$ such that $\Delta \circ \nabla=0$ on $\mathscr{E}$ then $\Delta=0$ on $\mathscr{E}$ as well, and the same holds true with $\nabla^{*}$ instead of $\nabla$.

An evolutionary field $E_{\varphi}$ is a symmetry of the equation $\mathscr{E}$ if $\left.E_{\varphi}(F)\right|_{\mathscr{E}}=\ell_{\mathscr{E}}(\varphi)=$ 0 . If $E_{\varphi}$ is a symmetry then $\varphi$ is said to be its generating function. We often identify symmetries with their generating functions.

A vector function $S=\left(S^{1}, \ldots, S^{n}\right)$ on $\mathscr{E}$ is a conserved current if $\sum_{i} D_{i}\left(S^{i}\right)=0$ on $\mathscr{E}$. A conserved current is trivial if there exist functions $T_{i j}$ on $\mathscr{E}$ such that $S^{i}=$ $\sum_{j<i} D_{j}\left(T^{j i}\right)-\sum_{i<j} D_{j}\left(T^{i j}\right)$. 
Conservation laws of $\mathscr{E}$ are classes of conserved currents modulo trivial ones. To every conservation law, there correspond its generating function, which is computed in the following way. If $S=\left(S^{1}, \ldots, S^{n}\right)$ is a conserved current, so that $\sum_{i} D_{i}\left(S^{i}\right)=0$ on $\mathscr{E}$, then there exists a $\mathscr{C}$-differential operator $\Delta$ such that $\sum_{i} D_{i}\left(S^{i}\right)=$ $\Delta(F)$ on $J^{\infty}$. The generating function of the conservation law is defined by $\psi=$ $\left(\psi_{1}, \ldots, \psi_{m}\right)=\Delta^{*}(1)$. Note that $\psi=0$ if and only if the conserved current $S$ is trivial. One can prove that every generating function $\psi$ satisfies the equation $\ell_{\mathscr{E}}^{*}(\psi)=0$, so that the set $\operatorname{CL}(\mathscr{E})$ of conservation laws of $\mathscr{E}$ is a subset in the kernel of $\ell_{\mathscr{E}}^{*}$, $\mathrm{CL}(\mathscr{E}) \subset \operatorname{ker} \ell_{\mathscr{E}}^{*}$.

\section{Cotangent bundle to an equation}

Let us introduce our main hero. For every differential equation $\mathscr{E}$ we define a canonical covering $\tau^{*}: \mathscr{L}^{*}(\mathscr{E}) \rightarrow \mathscr{E}$, called the $\ell^{*}$-covering. The equation $\mathscr{L}^{*}(\mathscr{E})$ is given by the system

$$
\ell_{F}^{*}(p)=0, \quad F=0,
$$

if $\mathscr{E}$ is given by $F=0$. Here $p=\left(p^{1}, \ldots, p^{l}\right)$ are new dependent variables, $l$ being the number of equations $F=\left(F_{1}, \ldots, F_{l}\right)$. We endow $\mathscr{L}^{*}(\mathscr{E})$ with the structure of a supermanifold by choosing the variables $p^{k}$ to be odd. The covering $\tau^{*}$ is the natural projection $\tau^{*}:\left(u_{\sigma}^{j}, p_{\sigma}^{k}\right) \mapsto\left(u_{\sigma}^{j}\right)$.

Note that

$$
\langle F, p\rangle=\sum_{i=1}^{l} F_{i} p^{i}
$$

is the Lagrangian for the equation $\mathscr{L}^{*}(\mathscr{E})$.

It is easily shown that $\ell_{\mathscr{L} *}(\mathscr{E})$ is normal if $\ell_{\mathscr{E}}$ is normal.

From the above definition it is not seen why we said that $\ell^{*}$-covering is canonical. Indeed, the definition uses the embedding $\mathscr{E} \rightarrow J^{\infty}$, but later we will show that $\mathscr{L}^{*}(\mathscr{E})$ is independent of the choice of this embedding.

Remark 1. For an arbitrary $\mathscr{C}$-differential operator $\Delta$ one can define the $\Delta$-covering in the same way as the $\ell^{*}$-covering is associated with the operator $\ell_{\mathscr{E}}^{*}$.

The most interesting for us property of the $\ell^{*}$-covering is given by the following theorem.

Theorem 1. There is a natural 1-1 correspondence between the symmetries of $\mathscr{E}$ and the conservation laws of $\mathscr{L}^{*}(\mathscr{E})$ linear along the fibers of $\tau^{*}$.

The expression "linear conservation law" means that the corresponding conserved current is linear along the fibers of $\tau^{*}$ (i.e., linear in variables $p^{k}$ ). Here and below we skip the proofs that can be found in our joint paper with S. Igonin [3]. Let us nevertheless describe the correspondence stated in the theorem in terms of generating functions. If $\varphi$ is a symmetry of equation $\mathscr{E}$ then there exists a $\mathscr{C}$-differential 
operator $\Delta$ such that $\ell_{F}(\varphi)=\Delta(F)$. Consider the adjoint operator $\Delta^{*}$. It can be naturally identified with a fiberwise linear vector function $\varphi_{\Delta}$ on $\mathscr{L}^{*}(\mathscr{E})$. Then the vector function $\left(\varphi, \varphi_{\Delta}\right)$ is the generating function of the conservation law that corresponds to the symmetry $\varphi$.

In the geometry of differential equation it is very useful to construct an analogy with geometry of finite dimensional manifolds. We shall now use this approach to clarify the meaning of the above theorem. Let us start building our analogy with the following two rather standard correspondences (cf. [10] and references therein):

\section{Manifold $M \quad$ PDE $\mathscr{E}$ \\ functions $\longleftrightarrow$ conservation laws \\ vector fields $\longleftrightarrow$ symmetries}

Now, using Theorem 1, we can say that the analog of the $\ell^{*}$-covering is a vector bundle such that vector fields on the base are in 1-1 correspondence with fiberwise linear functions on the total space of the bundle. Obviously, such a bundle is the cotangent bundle. So, the $\ell^{*}$-covering is the cotangent bundle to an equation, and we can continue our manifold-equation dictionary:

$\begin{aligned} \text { Manifold } M & \text { PDE } \mathscr{E} \\ \text { functions } & \longleftrightarrow \text { conservation laws } \\ \text { vector fields } & \longleftrightarrow \text { symmetries } \\ T^{*}(M) & \longleftrightarrow \mathscr{L}^{*}(\mathscr{E})\end{aligned}$

Remark 2. This dictionary can be easily extended:

$$
\begin{aligned}
\text { Manifold } M & \text { PDE } \mathscr{E} \\
\text { functions } & \longleftrightarrow \text { conservation laws } \\
\text { vector fields } & \longleftrightarrow \text { symmetries } \\
T^{*}(M) & \longleftrightarrow \mathscr{L}^{*}(\mathscr{E}) \\
T(M) & \left.\longleftrightarrow \mathscr{L}_{\mathscr{E}}\right)
\end{aligned}
$$

De Rham complex $\longleftrightarrow E_{1}^{0, n-1} \rightarrow E_{1}^{1, n-1} \rightarrow E_{1}^{2, n-1} \rightarrow \cdots$

Here $\mathscr{L}(\mathscr{E})$ is the $\ell$-covering (see Remark 1). The complex $E_{1}^{0, n-1} \rightarrow E_{1}^{1, n-1} \rightarrow$ $E_{1}^{2, n-1} \rightarrow \cdots$ is $(n-1)$ st line of the Vinogradov $\mathscr{C}$-spectral sequence (see [10] and references therein). In this paper we use only the first three entries of the dictionary.

Remark 3. In [7], Kupershmidt defined the cotangent bundle to a bundle. This construction can be identified with the $\ell^{*}$-covering of the system

$$
u_{t}^{1}=0, \quad u_{t}^{2}=0, \quad \ldots \quad u_{t}^{m}=0 .
$$


At this point, a natural question may arise: what is the analog of the Poisson bracket on the cotangent bundle? The answer is that the $\ell^{*}$-covering is endowed with a canonical Poisson bracket. More precisely, since we changed the parity of fibers in the $\ell^{*}$-covering, this bracket is a superbracket and is the analog of the Schouten bracket. We shall call it the variational Schouten bracket.

To define the bracket, recall that $\mathscr{L}^{*}(\mathscr{E})$ has the Lagrangian structure (7). Hence, by the Noether theorem there is a 1-1 correspondence between conservation laws on $\mathscr{L}^{*}(\mathscr{E})$ and Noether symmetries of $\mathscr{L}^{*}(\mathscr{E})$. If $\psi$ is the generating function of a conservation law, then $E_{\psi}$ is the corresponding Noether symmetry. The set of Noether symmetries is a Lie superalgebra with respect to the commutator, so we obtain a structure of Lie superalgebra on conservation laws on $\mathscr{L}^{*}(\mathscr{E})$ uniquely determined by the equality

$$
E_{\llbracket \psi_{1}, \psi_{2} \rrbracket}=\left[E_{\psi_{1}}, E_{\psi_{2}}\right] .
$$

According to our manifold-equation dictionary, conservation laws on $\mathscr{L}^{*}(\mathscr{E})$ correspond to functions on $T^{*}(M)$. The latter are skew multivectors on $M$ (this is why we have changed the parity of fibers of the $\ell^{*}$-covering-to get skew-symmetric multivectors). So, we shall call conservation laws on $\mathscr{L}^{*}(\mathscr{E})$ the variational multivectors. Linear conservation laws, as we saw, are vectors, biliner ones are bivectors and so on.

The generating function of a variational $k$-vector is a vector function on $\mathscr{L}^{*}(\mathscr{E})$ which is $(k-1)$-linear along $\tau^{*}$-fibers. Such a function can be identified with a $(k-1)$-linear $\mathscr{C}$-differential operator on $\mathscr{E}$. In coordinates, this correspondence boils down to the change $p_{\sigma} \mapsto D_{\sigma}$. Thus, we can (and will) identify variational multivectors to multilinear $\mathscr{C}$-differential operators.

More precisely, in the above identification we will use not operators but equivalence classes of $\mathscr{C}$-differential operators modulo operators divisible by $\ell_{\mathscr{E}}^{*}$. This is being done, because operators of the form $\square \circ \ell_{\mathscr{E}}^{*}$ correspond to trivial functions on $\mathscr{L}^{*}(\mathscr{E})$. But we will not change terminology, we say operator instead of the equivalence class.

For the sake of brevity and because we are interested in the Hamiltonian formalism, let us restrict ourselves to bivectors, which are identified with linear $\mathscr{C}$ differential operators. Formulas presented below for bivectors (= linear operators) can be easily generalised to multivectors (= multilinear operators).

Theorem 2. An operator $A$ is a variational bivector on equation $\mathscr{E}$ if and only if it satisfies the condition

$$
\ell_{\mathscr{E}} A=A^{*} \ell_{\mathscr{E}}^{*} .
$$

Remark 4. If $\mathscr{E}$ is written in evolution form then the above condition implies that $A^{*}=-A$.

From this theorem it follows that a Hamiltonian operator $A$ takes a generating function of a conservation law $\psi$ to a symmetry $A(\psi)$.

This is the formula for the variational Schouten bracket of two bivectors: 


$$
\begin{aligned}
& {\left[A_{1}, A_{2}\right]\left(\psi_{1}, \psi_{2}\right)} \\
& =\ell_{A_{1}, \psi_{1}}\left(A_{2}\left(\psi_{2}\right)\right)-\ell_{A_{1}, \psi_{2}}\left(A_{2}\left(\psi_{1}\right)\right) \\
& +\ell_{A_{2}, \psi_{1}}\left(A_{1}\left(\psi_{2}\right)\right)-\ell_{A_{2}, \psi_{2}}\left(A_{1}\left(\psi_{1}\right)\right) \\
& \quad-A_{1}\left(B_{2}^{*}\left(\psi_{1}, \psi_{2}\right)\right)-A_{2}\left(B_{1}^{*}\left(\psi_{1}, \psi_{2}\right)\right),
\end{aligned}
$$

where $\ell_{A, \psi}=\ell_{A(\psi)}-A \ell_{\psi}$ and the operators $B_{i}^{*}$ are defined by the equalities:

$$
\begin{aligned}
\ell_{F} A_{i}-A_{i}^{*} \ell_{F}^{*} & =B_{i}(F, \cdot) \quad \text { on } J^{\infty}, \\
B_{i}^{*}\left(\psi_{1}, \psi_{2}\right) & =\left.B_{i}^{*}\left(\psi_{1}, \psi_{2}\right)\right|_{\mathscr{E}} .
\end{aligned}
$$

Here ${ }^{*} 1$ denotes that the adjoint operator is computed with respect to the first argument. The operators $B_{i}^{*}$ are skew-symmetric and skew-adjoint in each argument. Note that if $\mathscr{E}$ is in evolution form then $B_{i}^{*}\left(\psi_{1}, \psi_{2}\right)=\ell_{A_{i}, \psi_{2}}^{*}\left(\psi_{1}\right)$.

Now we are in position to give a definition of a Hamiltonian structure for a general PDE.

Definition 2. $A$ variational bivector $A$ is called Hamiltonian if $[[A, A]]=0$.

A Hamiltonian bivector $A$ gives rise to a Poisson bracket

$$
\left\{\psi_{1}, \psi_{2}\right\}_{A}=E_{A\left(\psi_{1}\right)}\left(\psi_{2}\right)+\Delta^{*}\left(\psi_{2}\right),
$$

where $\psi_{1}$ and $\psi_{2}$ are conservation laws of $\mathscr{E}$ and the operator $\Delta$ is defined by the relation $\ell_{F}\left(A\left(\psi_{1}\right)\right)=\Delta(F)$.

As in the evolution case, we call an equation bi-Hamiltonian if it possesses two Hamiltonian structures $A_{1}$ and $A_{2}$ such that $\left[\left[A_{1}, A_{2}\right]\right]=0$.

An infinite series of conservation laws $\psi_{1}, \psi_{2}, \ldots$ is called a Magri hierarchy if for all $i$ we have $A_{1}\left(\psi_{i}\right)=A_{2}\left(\psi_{i+1}\right)$. In the standard way one can show that $\left\{\psi_{i}, \psi_{j}\right\}_{A_{1}}=\left\{\psi_{i}, \psi_{j}\right\}_{A_{2}}=0$ for all $i$ and $j$.

Now let us return to the question of invariance of the $\ell^{*}$-covering. Suppose the equation $\mathscr{E}$ under consideration is embedded in two different jet spaces

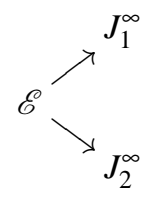

We encountered an example of this situation when discussed the KdV equation, with $J_{1}^{\infty}$ being jets with coordinates $x, t$ and $u$, while $J_{2}^{\infty}$ being jets with coordinates $x, t, u$, $v$, and $w$. Now, we have two linearization operators, $\ell_{\mathscr{E}}^{1}$ and $\ell_{\mathscr{E}}^{2}$, the former computed using the embedding $\mathscr{E} \rightarrow J_{1}^{\infty}$ and the latter is obtained using the embedding $\mathscr{E} \rightarrow J_{2}^{\infty}$. It is not difficult to show that these two linearization operators are related by the following diagram: 


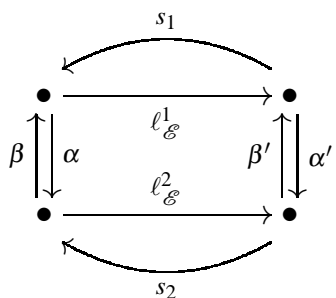

where all arrows are $\mathscr{C}$-differential operators on $\mathscr{E}$ satisfying the following relations:

$$
\ell_{\mathscr{E}}^{1} \beta=\beta^{\prime} \ell_{\mathscr{E}}^{2}, \quad \ell_{\mathscr{E}}^{2} \alpha=\alpha^{\prime} \ell_{\mathscr{E}}^{1}, \quad \beta \alpha=\mathrm{id}+s_{1} \ell_{\mathscr{E}}^{1}, \quad \alpha \beta=\mathrm{id}+s_{2} \ell_{\mathscr{E}}^{2} .
$$

We use the dots $\bullet$ to avoid introducing new notations for the corresponding spaces of sections of vector bundles.

Definition 3. Two $\mathscr{C}$-differential operators $\Delta_{1}$ and $\Delta_{2}$ on $\mathscr{E}$ are called equivalent if there exist $\mathscr{C}$-differential operators $\alpha, \beta, \alpha^{\prime}, \beta^{\prime}, s_{1}$, and $s_{2}$ such that

$$
\Delta_{1} \beta=\beta^{\prime} \Delta_{2}, \quad \Delta_{2} \alpha=\alpha^{\prime} \Delta_{1}, \quad \beta \alpha=\mathrm{id}+s_{1} \Delta_{1}, \quad \alpha \beta=\mathrm{id}+s_{2} \Delta_{2} .
$$

(see [2] and references therein). Thus, we can say that the linearization operators $\ell_{\mathscr{E}}^{1}$ and $\ell_{\mathscr{E}}^{2}$ are equivalent.

The following simple Lemma explains why this notion is really important.

Lemma 1. $\mathscr{C}$-differential operators $\Delta_{1}$ and $\Delta_{2}$ are equivalent if and only if the $\Delta_{1}$ and $\Delta_{2}$-coverings are isomorphic as linear coverings.

So, to prove that $\ell^{*}$-covering is invariant we have to establish that the operators $\ell_{\mathscr{E}}^{1 *}$ and $\ell_{\mathscr{E}}^{2 *}$ are equivalent. This is implied by the following result.

Theorem 3. If two normal operators $\Delta_{1}$ and $\Delta_{2}$ are equivalent then $\Delta_{1}^{*}$ is equivalent to $\Delta_{2}^{*}$.

Corollary 1. The equation $\mathscr{L}^{*}(\mathscr{E})$ does not depend on the embedding $\mathscr{E} \rightarrow J^{\infty}$.

Now, recall that bivectors were defined as conservation laws on $\mathscr{L}^{*}(\mathscr{E})$, while operators that correspond to them are essentially generating functions of these conservation laws. Thus, the operators depend on using an embedding $\mathscr{E} \rightarrow J^{\infty}$. Assume that we have two different embeddings as above, so that they give rise to two operators $A^{1}$ and $A^{2}$ that correspond to the same bivector. Here are the formulas that relate these two operators:

$$
\begin{aligned}
& A^{2}=\alpha A^{1} \alpha^{*}, \\
& A^{1}=\beta A^{2} \beta^{\prime *} .
\end{aligned}
$$




\section{Examples}

Let us revise the three examples from the Introduction.

Example $4(K d V)$. We considered two different embeddings of the $\mathrm{KdV}$ equation to jets:

$$
\begin{gathered}
u_{t}-u_{x x x}-6 u u_{x}=0, \\
\left(\begin{array}{c}
u_{x}-v \\
v_{x}-w \\
w_{x}-u_{t}+6 u v
\end{array}\right)=0 .
\end{gathered}
$$

Here are all operators of diagram (10):

$$
\begin{aligned}
& \ell_{\mathscr{E}}^{1}=D_{t}-D_{x x x}-6 u D_{x}-6 u_{x}, \quad \ell_{\mathscr{E}}^{2}=\left(\begin{array}{ccc}
D_{x} & -1 & 0 \\
0 & D_{x} & -1 \\
-D_{t}+6 v & 6 u & D_{x}
\end{array}\right), \\
& \alpha=\left(\begin{array}{c}
1 \\
D_{x} \\
D_{x x}
\end{array}\right), \quad \alpha^{\prime}=\left(\begin{array}{r}
0 \\
0 \\
-1
\end{array}\right), \quad \begin{array}{lll}
\beta & =\left(\begin{array}{lll}
1 & 0 & 0
\end{array}\right), \\
\beta^{\prime}=\left(\begin{array}{lll}
-D_{x x}-6 u & -D_{x} & -1
\end{array}\right),
\end{array} \\
& s_{1}=0, \quad s_{2}=\left(\begin{array}{ccc}
0 & 0 & 0 \\
1 & 0 & 0 \\
D_{x} & 1 & 0
\end{array}\right) .
\end{aligned}
$$

Formulas (12) relate Hamiltonian operators (1) and (3).

Remark 5. If we take an operator from (1) for $A^{1}$ and compute $A^{2}$ via (12) we will get an operator from (3) only up to the equivalence.

Example 5 (Camassa-Holm equation). The Camassa-Holm equation written in the usual form $u_{t}-u_{t x x}-u u_{x x x}-2 u_{x} u_{x x}+3 u u_{x}=0$ has a bi-Hamiltonian structure:

$$
A_{1}=D_{x} \quad A_{2}=-D_{t}-u D_{x}+u_{x} .
$$

If we rewrite the equation in the form

$$
\begin{aligned}
& m_{t}+u m_{x}+2 u_{x} m=0, \\
& m-u+u_{x x}=0
\end{aligned}
$$

then the bi-Hamiltonian structure takes the form

$$
A_{1}^{\prime}=\left(\begin{array}{cc}
D_{x} & 0 \\
D_{x}-D_{x}^{3} & 0
\end{array}\right) \quad A_{2}^{\prime}=\left(\begin{array}{cc}
0 & -1 \\
2 m D_{x}+m_{x} & 0
\end{array}\right)
$$

Note that the operators $B_{1}$ and $B_{2}$ from Example 2 are entries (up to sign) of the matrix $A_{1}^{\prime}$ and $A_{2}^{\prime}$. Thus we see that studying bi-Hamiltonian structure of the CamassaHolm equation does not require the use of the $\left(1-D_{x}^{2}\right)^{-1}$ "operator". 
Example 6 (Kupershmidt deformation). Let $\mathscr{E}$ be a bi-Hamiltonian equation given by $F=0$ and $A_{1}$ and $A_{2}$ be the Hamiltonian operators.

Definition 4. The Kupershmidt deformation $\tilde{\mathscr{E}}$ of $\mathscr{E}$ has the form

$$
F+A_{1}^{*}(w)=0, \quad A_{2}^{*}(w)=0,
$$

where $w=\left(w^{1}, \ldots, w^{l}\right)$ are new dependent variables.

Theorem 4. The Kupershmidt deformation $\tilde{\mathscr{E}}$ is a bi-Hamiltonian system.

The proof of this theorem consists of checking that the following two bivectors define a bi-Hamiltonian structure:

$$
\tilde{A}_{1}=\left(\begin{array}{cc}
A_{1} & -A_{1} \\
0 & \ell_{F+A_{1}^{*}(w)+A_{2}^{*}(w)}
\end{array}\right) \quad \tilde{A}_{2}=\left(\begin{array}{cc}
A_{2} & -A_{2} \\
-\ell_{F+A_{1}^{*}(w)+A_{2}^{*}(w)} & 0
\end{array}\right)
$$

The generalisation of Kupershmidt's theorem from the Introduction is the following.

Theorem 5. If $\psi_{1}, \psi_{2}, \ldots$ is a Magri hierarchy for $\mathscr{E}$ then, under some technical assumptions, $\left(\psi_{i},-\psi_{i+1}\right), i=1,2, \ldots$, is a Magri hierarchy for the Kupershmidt deformation $\tilde{\mathscr{E}}$.

Details and proofs of Theorem 4 and 5 can be found in [5].

Acknowledgement. We wish to thank the organizers and participants of the Abel Symposium 2008 in Troms $\emptyset$ for making the conference a productive and enlivening event. We also are grateful to Sergey Igonin for reading a draft of this paper and useful comments.

\section{References}

1. Bocharov, A.V., Chetverikov, V.N., Duzhin, S.V., Khor'kova, N.G., Krasil'shchik, I.S., Samokhin, A.V., Torkhov, Y.N., Verbovetsky, A.M., Vinogradov, A.M.: Symmetries and Conservation Laws for Differential Equations of Mathematical Physics. Monograph. Amer. Math. Soc. (1999)

2. Dudnikov, P., Samborski, S.: Linear overdetermined systems of partial differential equations. In: M. Shubin (ed.) Partial Differential Equations VIII, Encycl. Math. Sci., vol. 65, pp. 1-86. Springer-Verlag (1996)

3. Igonin, S., Kersten, P., Krasilshchik, I., Verbovetsky, A., Vitolo, R.: Variational brackets in the geometry of PDEs (2009). To appear

4. Karasu-Kalkanlı, A., Karasu, A., Sakovich, A., Sakovich, S., Turhan, R.: A new integrable generalization of the Korteweg-de Vries equation. J. Math. Phys. 49, 073,516 (2008), arXiv: 0708.3247

5. Kersten, P., Krasil'shchik, I., Verbovetsky, A., Vitolo, R.: Integrability of Kupershmidt deformations. Acta Appl. Math. (2009). To appear

6. Krasil'shchik, J., Verbovetsky, A.M.: Homological Methods in Equations of Mathematical Physics. Advanced Texts in Mathematics. Open Education \& Sciences, Opava (1998), arXiv:math/9808130 
7. Kupershmidt, B.A.: Geometry of jet bundles and the structure of Lagrangian and Hamiltonian formalism. In: G. Kaiser, J.E. Marsden (eds.) Geometric Methods in Mathematical Physics, Lect. Notes in Math., pp. 162-218. Springer-Verlag (1980)

8. Kupershmidt, B.A.: KdV6: An integrable system. Phys. Lett. A 372, 2634-2639 (2008), arXiv:0709.3848

9. Tsarev, S.: The Hamilton property of stationary and inverse equations of condensed matter mechanics and mathematical physics. Math. Notes 46, 569-573 (1989)

10. Vinogradov, A.M.: Cohomological Analysis of Partial Differential Equations and Secondary Calculus. Amer. Math. Soc. (2001) 